\title{
Gaussian and non-Gaussian fluctuations in pure classical fluids
}

Nawavi Naleem, , Elizabeth A. Ploetz, and, and Paul E. Smith

Citation: The Journal of Chemical Physics 146, 094509 (2017); doi: 10.1063/1.4977455

View online: http://dx.doi.org/10.1063/1.4977455

View Table of Contents: http://aip.scitation.org/toc/jcp/146/9

Published by the American Institute of Physics

\section{Articles you may be interested in}

Fluctuation solution theory of pure fluids

The Journal of Chemical Physics 146, 094501 (2017); 10.1063/1.4977040

Perspective: Dissipative particle dynamics

The Journal of Chemical Physics 146, 150901 (2017); 10.1063/1.4979514

The cage effect in systems of hard spheres

The Journal of Chemical Physics 146, 104503 (2017); 10.1063/1.4977523

Announcement: Top reviewers for The Journal of Chemical Physics 2016

The Journal of Chemical Physics 146, 100201 (2017); 10.1063/1.4978399

A Gaussian theory for fluctuations in simple liquids

The Journal of Chemical Physics 146, 134507 (2017); 10.1063/1.4979659

Temperature fluctuations and the thermodynamic determination of the cooperativity length in glass forming liquids

The Journal of Chemical Physics 146, 104501 (2017); 10.1063/1.4977737

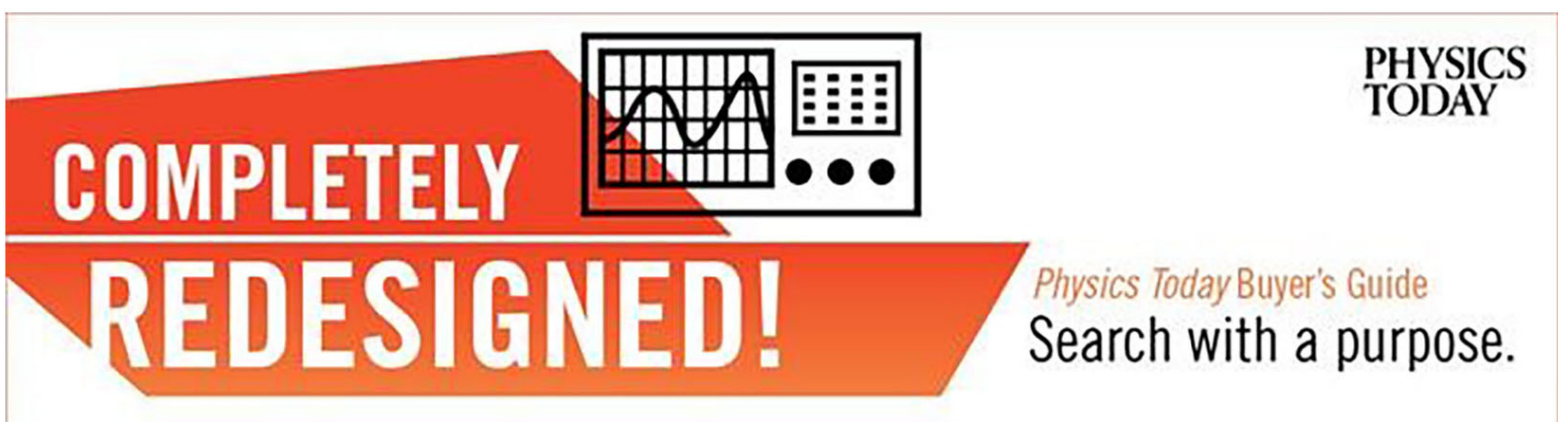




\title{
Gaussian and non-Gaussian fluctuations in pure classical fluids
}

\author{
Nawavi Naleem, Elizabeth A. Ploetz, ${ }^{\text {a) }}$ and Paul E. Smith \\ Department of Chemistry, Kansas State University, 213 CBC Building, 1212 Mid Campus Dr. North, Manhattan, \\ Kansas 66506-0401, USA
}

(Received 21 November 2016; accepted 12 February 2017; published online 6 March 2017)

\begin{abstract}
The particle number, energy, and volume probability distributions in the canonical, isothermalisobaric, grand canonical, and isobaric-isenthalpic ensembles are investigated. In particular, we consider Gaussian and non-Gaussian behavior and formulate the results in terms of a single expression valid for all the ensembles employing common, experimentally accessible, thermodynamic derivatives. This is achieved using Fluctuation Solution Theory to help manipulate derivatives of the entropy. The properties of the distributions are then investigated using available equations of state for fluid water and argon. Purely Gaussian behavior is not observed for any of the state points considered here. A set of simple measures, involving thermodynamic derivatives, indicating non-Gaussian behavior is proposed. A general expression, valid in the high temperature limit, for small energy fluctuations in the canonical ensemble is provided. Published by AIP Publishing. [http://dx.doi.org/10.1063/1.4977455]
\end{abstract}

\section{INTRODUCTION}

Most applications of equilibrium statistical mechanics focus on the determination of ensemble averages, e.g., pressure, density, and chemical potential, that correspond to first derivatives of the thermodynamic potentials. ${ }^{1}$ In the thermodynamic limit, the same results are then obtained for all ensembles. In contrast, fluctuations are often only used to illustrate that the width of the energy distribution, for a system in the canonical ensemble, for example, is negligible when compared to the mean energy of the system. ${ }^{1,2}$ However, this implies that fluctuations are relatively unimportant. This is not true and many thermodynamic properties can be related to the fluctuations, although the fluctuations then depend on the ensemble of interest, i.e., the thermodynamic constraints placed on the system. In particular, the second moments of the corresponding probability distributions can be directly related to second derivatives of the thermodynamic potentials - and include the important response functions. ${ }^{2,3}$

The study of equilibrium fluctuations has a long history and the interested reader is referred to a comprehensive review by Ruppeiner for more details. ${ }^{4}$ Equilibrium fluctuations can be related to a series of thermodynamic properties (derivatives), and play an integral role in a variety of fluctuationdissipation theorem based relationships, ${ }^{5,6}$ and other fluid behaviors. ${ }^{7,8}$ The majority of approaches involve an expansion of the entropy. The most common treatment of fluctuations is provided by Landau and Lifshitz, ${ }^{9}$ while a more extensive discussion was recently provided by Mishin. ${ }^{10}$ However, it is rare to expand beyond second order, which limits the resulting probability distribution to be Gaussian in nature. This is the distribution often expected in the thermodynamic limit. ${ }^{4}$ Ruppeiner and others have indicated that there are significant problems with (energy) conservation and non-covariant

\footnotetext{
a) Present address: Johns Hopkins University School of Medicine, $725 \mathrm{~N}$. Wolfe St., Baltimore, MD 21205, USA.
}

behavior of entropy expansions beyond the Gaussian approximation. ${ }^{4,11,12}$ Nevertheless, there is interest in the properties of the resulting non-Gaussian distributions. ${ }^{4,12-14}$ The third and higher moments of these distributions are finite and have been developed by Greene and Callen. ${ }^{15}$ They are important for understanding higher thermodynamic derivatives, especially close to the critical point, which characterize the behavior of fluids and their mixtures. Unfortunately, knowing the moments does not necessarily lead to the underlying distribution.

In principle, one can directly measure the fluctuations experimentally. For instance, particle number (density) fluctuations in fluids can be measured using light scattering. ${ }^{16,17}$ It is unlikely, however, that these methods can be easily extended to provide higher moments of the distributions characterizing any non-Gaussian behavior. Alternatively, the fluctuations can be directly related to thermodynamic derivatives and properties of fluids that are relatively easy to determine. In the Gaussian approximation, the distributions are characterized by their covariance, corresponding to fluctuations and cross fluctuations between pairs of variables, and the covariance matrix is then directly related to second derivatives of the thermodynamic potentials. ${ }^{2,10}$ Non-Gaussian behavior is then manifested in the deviations from Gaussian behavior characterized by the coskewness of the distribution, corresponding to correlations between three variables, and can be directly related to third derivatives of the thermodynamic potentials. ${ }^{15}$ This is the approach taken here.

Recently, we have been using Fluctuation Solution Theory (FST) to investigate the properties of liquids and liquid mixtures. ${ }^{14,18-20}$ FST provides an alternative view of the commonly studied closed system thermodynamic properties in terms of fluctuating quantities occurring in an equivalent Grand Canonical Ensemble (GCE) system. FST represents a generalization of the Kirkwood-Buff theory of solutions, ${ }^{21-23}$ to include energy fluctuations. ${ }^{19}$ By examining the corresponding fluctuations, one can obtain further insight into the properties of mixtures and simple fluids. ${ }^{14,24}$ It is well known that 
particle and energy pair fluctuations in the GCE can be related to the response functions. However, it is less common to use the particle-energy triplet (or higher) fluctuations to investigate higher derivatives of the response functions. Nevertheless, the third moments of the bivariate particle-energy joint distribution in the GCE can be useful and quantify the non-Gaussian behavior of the underlying probability distribution. ${ }^{14}$

Here, we use FST to help provide the second and third derivatives of the entropy for use in the usual entropy expansion to provide a general non-Gaussian particle, volume, and energy probability distribution that is applicable to any ensemble. The initial steps essentially follow previous entropy expansion approaches, ${ }^{9,15}$ although here we start from the probability distributions for a series of ensembles. These general expressions are then developed, using FST, to provide the Gaussian and non-Gaussian distributions in terms of common thermodynamic derivatives available experimentally. The nature of the resulting distribution is then investigated.

\section{THEORY}

\section{A. Fluctuations}

The thermodynamic potentials associated with the four main ensembles of statistical mechanics can be written as ${ }^{1,2}$

$$
\begin{aligned}
S / R & =\ln \Omega\left(N_{1}, V, E\right), \\
-\beta A & =-\beta\langle E\rangle+\langle S\rangle / R=\ln Q\left(N_{1}, V, T\right), \\
-\beta G & =-\beta\langle E\rangle-\beta p\langle V\rangle+\langle S\rangle / R=\ln \Delta\left(N_{1}, p, T\right), \\
\beta p V & =-\beta\langle E\rangle+\beta \mu_{1}\left\langle N_{1}\right\rangle+\langle S\rangle / R=\ln \Xi\left(\mu_{1}, V, T\right),
\end{aligned}
$$

where $\beta=1 /(R T)$ and $R$ is the gas constant. The symbols $\Omega$, $Q, \Delta$, and $\Xi$ represent the partition functions for the microcanonical (NVE), canonical $(N V T)$, isothermal-isobaric/Gibbs $(N p T)$, and grand canonical $(\mu V T)$ ensembles, respectively. These are then related to the number of particles $\left(N_{1}\right)$, the volume $(V)$, the internal energy $(E)$, the entropy $(S)$, the pressure $(p)$, the absolute temperature $(T)$, the chemical potential $\left(\mu_{1}\right)$, the Helmholtz free energy $(A)$, and the Gibbs free energy $(G)$ of the system. The angular brackets denote the appropriate ensemble averages, and it is understood that for the entropy $\langle S\rangle=S\left(\left\langle N_{1}\right\rangle,\langle V\rangle,\langle E\rangle\right)$. Formally, the entropy described above corresponds to an entropy maximum and therefore the average internal energy, $\langle E\rangle$, should really be the most probable internal energy, $E^{*}$. However, we can assume these to be equivalent in the thermodynamic limit (see later discussion).

The conditional probability that a system has a particular energy, volume, and/or particle number between $E$ and $E+d E$, etc., is then given by

$$
\begin{aligned}
P\left(E \mid N_{1}, V\right) d E & =\frac{e^{-\beta E} e^{S / R}}{Q} d E=e^{-\beta \delta E+\delta S / R} d E, \\
P\left(V, E \mid N_{1}\right) d V d E & =\frac{e^{-\beta E} e^{-\beta p V} e^{S / R}}{\Delta} d V d E \\
& =e^{-\beta \delta E-\beta p \delta V+\delta S / R} d V d E, \\
P\left(N_{1}, E \mid V\right) d N_{1} d E & =\frac{e^{-\beta E} e^{\beta \mu_{1} N_{1}} e^{S / R}}{\Xi} d N_{1} d E \\
& =e^{-\beta \delta E+\beta \mu_{1} \delta N_{1}+\delta S / R} d N_{1} d E
\end{aligned}
$$

for the $N V T, N p T$, and $\mu V T$ ensembles, respectively. Here, $\delta X=X-\langle X\rangle$ represents a fluctuation in the property $X=N_{1}$, $V, E$, or $S$. Clearly, a general probability distribution function (pdf) can be written as

$$
\ln P\left(N_{1}, V, E\right)=\beta \mu_{1} \delta N_{1}-Z \rho_{1} \delta V-\beta \delta E+\delta S / R,
$$

where $\rho_{1}=N_{1} / V$ is the number density, $Z=\beta p V_{1}$ is the compressibility factor of the pure fluid, and $V_{1}$ is the molar volume. This expression is applicable to all the ensembles, with the understanding that the ensembles provide the same ensemble average thermodynamic properties, and that $\delta N_{1}$ is zero in a closed system, etc.

The presence of the entropy in Equation (3) is problematic. In order to develop this expression further, knowledge of the variation in entropy (density of states) with the energy, volume, and/or particle number is required. ${ }^{15}$ The entropy variations can be evaluated by noting that a Taylor expansion of the entropy around $\langle E\rangle=E^{*}$, etc., provides

$$
\delta S / R=S^{(1)}+\frac{1}{2} S^{(2)} N_{1}^{-1}+\frac{1}{6} S^{(3)} N_{1}^{-2}+\cdots,
$$

where the $N_{1}$ 's have been included to make the corresponding entropy derivatives that appear in the $S^{(\mathrm{n})}$ terms intensive. We will assume that these derivatives exist and that the expansion is valid, which is generally true if we stay away from phase boundaries and the critical point. The $S^{(\mathrm{n})}$ 's describe a series of increasingly more complicated terms given by

$$
\begin{aligned}
S^{(1)} & =\sum_{x} S_{x} \delta x, \\
S^{(2)} & =\sum_{x, y} S_{x y} \delta x \delta y, \\
S^{(3)} & =\sum_{x, y, z}^{y} S_{x y z} \delta x \delta y \delta z,
\end{aligned}
$$

where $x, y$, or $z$ refer to each of the three dimensionless fluctuations given by $\beta E, \rho_{1} V$, or $N_{1}$, corresponding to derivatives of the entropy with respect to $E, V$, or $N_{1}$. Here we will only consider the terms up to and including third derivatives of the entropy. The sums in $S^{(1)}, S^{(2)}$, and $S^{(3)}$ involve 3, 9, and 27 terms with 3,6 , and 10 unique entropy derivatives $\left(S_{x}\right.$, $\left.S_{x y}, S_{x y z}\right)$, respectively. The exact form of the dimensionless intensive entropy derivatives is described in Appendix A.

The first and second law for an open system at equilibrium with only pressure-volume work can be written as ${ }^{25}$

$$
d S / R=-\beta \mu_{1} d N_{1}+Z \rho_{1} d V+\beta d E .
$$

This provides the first derivatives of the entropy given in Appendix A. Using these in Equations (3) and (4) leads to the general expression

$$
\ln P\left(N_{1}, V, E\right)=\frac{1}{2} S^{(2)} N_{1}^{-1}+\frac{1}{6} S^{(3)} N_{1}^{-2}+\cdots .
$$

Truncation after the $S^{(2)}$ term results in a Gaussian distribution, and represents the stopping point for many studies of fluctuations, while inclusion of the $S^{(3)}$ (and higher) term will lead to non-Gaussian distributions. The resulting probabilities are not normalized but it is clear that $P\left(\left\langle N_{1}\right\rangle,\langle V\rangle,\langle E\rangle\right)=1$, irrespective of how many terms are included in Equation (7). It should be noted that, using an entropy expansion approach beyond Gaussian, it is problematic to assume that the mean values 
of $N_{1}, V$, and $E$ are also the most probable values. Indeed, previous studies have shown that the GCE distributions are not Gaussian (or symmetric) and therefore this assumption is not justified for the present purposes. ${ }^{14,20}$ This point will also be discussed further in Sec. III.

In order to develop Equations (5) and (7), one requires higher derivatives of the entropy. To evaluate these second and third derivatives, we turn to FST. We will see below that not all these derivatives are available experimentally. Hence, further manipulation of the derivatives will also be required.

\section{B. Fluctuation Solution Theory}

The next step requires the conversion of the entropy derivatives to more common thermodynamic derivatives or combinations of derivatives. In our opinion, the second, and especially the third, derivatives can be evaluated most easily using a FST based approach utilizing the corresponding pair and triplet fluctuations for the equivalent GCE. To achieve this, we define a series of fluctuating pair and triplet densities

$$
\begin{aligned}
B_{X Y} & \equiv\langle\delta X \delta Y\rangle / V, \\
C_{X Y Z} & \equiv\langle\delta X \delta Y \delta Z\rangle / V,
\end{aligned}
$$

where $X, Y, Z=N_{1}$ (indicated by a subscript 1), $E, \varepsilon$, or $E^{\prime}$. The angular brackets denote a GCE average. While other fluctuating quantities will appear later, the most useful energy quantities for our initial manipulations are

$$
\begin{aligned}
\varepsilon & \equiv E-N_{1} H_{1}, \\
E^{\prime} & =E-N_{1} E_{1},
\end{aligned}
$$

where $\varepsilon$ and $E^{\prime}$ are the excess energies, $E$ and $N_{1}$ are the instantaneous (in the time average sense) internal energy and particle number for the equivalent GCE system, $H_{1}$ is the average molar enthalpy, and $E_{1}$ is the average molar internal energy. The main reason for adopting the above combinations is to negate the fact that the energy fluctuations in the GCE are sensitive to the zero of energy. ${ }^{24}$ The above pair fluctuation densities can be converted to a set of corresponding dimensionless counterparts (indicated by lower case letters) by

$$
\begin{aligned}
& b_{11} \equiv B_{11} / \rho_{1}, \\
& b_{1 \varepsilon} \equiv \beta B_{1 \varepsilon} / \rho_{1}, \\
& b_{\varepsilon \varepsilon} \equiv \beta^{2} B_{\varepsilon \varepsilon} / \rho_{1} .
\end{aligned}
$$

Similar expressions can be written for the $C$ 's and then provide the corresponding dimensionless $c$ 's. The above fluctuating quantities are related to common thermodynamic properties of fluids via ${ }^{18,19,26,27}$

$$
\begin{aligned}
\frac{p \kappa_{T}}{Z} & \equiv \frac{p}{\rho_{1} Z}\left(\frac{\partial \rho_{1}}{\partial p}\right)_{T}=b_{11}, \\
T \alpha_{p} & \equiv-\frac{T}{\rho_{1}}\left(\frac{\partial \rho_{1}}{\partial T}\right)_{p}=-b_{1 \varepsilon}, \\
\frac{C_{p, m}}{R} & \equiv \frac{1}{R}\left(\frac{\partial H_{1}}{\partial T}\right)_{p}=b_{\varepsilon \varepsilon}, \\
\frac{C_{V, m}}{R} & \equiv \frac{1}{R}\left(\frac{\partial E_{1}}{\partial T}\right)_{\rho_{1}}=b_{\varepsilon \varepsilon}-\frac{b_{1 \varepsilon}^{2}}{b_{11}},
\end{aligned}
$$

where $\kappa_{T}$ is the isothermal compressibility, $\alpha_{p}$ is the thermal expansion coefficient, $C_{p, m}$ is the isobaric molar heat capacity, and $C_{V, m}$ is the isochoric molar heat capacity. Hence, if one knows the $b$ 's for the GCE system, one also knows the response functions for the equivalent closed system. Likewise, if one knows the response functions, one also knows the pair fluctuations for an equivalent open GCE system. Finally, we note that the following combination of terms occurs repeatedly for the entropy derivatives and so we define

$$
S_{0} \equiv \frac{1}{b_{\varepsilon \varepsilon} b_{11}-b_{1 \varepsilon}^{2}}=\frac{Z}{p \kappa_{T}} \frac{R}{C_{V, m}}
$$

to help simplify many of the resulting equations. This particular combination of $B$ 's can be written in several ways

$$
B_{\varepsilon \varepsilon} B_{11}-B_{1 \varepsilon}^{2}=B_{E E} B_{11}-B_{1 E}^{2}=B_{E^{\prime} E^{\prime}} B_{11}-B_{1 E^{\prime}}^{2}
$$

which also helps to simplify the process of obtaining many of the third derivatives.

Using the above relationships, the six unique dimensionless second derivatives of the entropy are given by (see Appendix B)

$$
\begin{array}{lll}
S_{N N}=-S_{0} b_{E E}, & S_{V V}=-S_{0} b_{E^{\prime} E^{\prime}}, & S_{E E}=-S_{0} b_{11}, \\
S_{N V}=S_{0} b_{E E^{\prime}}, & S_{N E}=S_{0} b_{1 E}, & S_{V E}=-S_{0} b_{1 E^{\prime}}
\end{array}
$$

in terms of the dimensionless particle and energy fluctuations. It should be noted that $S_{N N}, S_{N V}$, and $S_{N E}$, i.e., the particle number derivatives, are not available experimentally as the energy fluctuations in the GCE depend on the zero of energy. The fluctuations that involve just $E^{\prime}$ do not depend on the zero of energy and hence $S_{V V}, S_{V E}$, and $S_{E E}$ are available.

The third derivatives of the entropy are more involved. They can be obtained from Equation (14) using the approach outlined in Appendix C. There are ten unique derivatives. In their most condensed form, they are given by

$$
\begin{aligned}
& S_{N N N}=-S_{0}^{3} c_{n n n}, \\
& S_{N N V}=S_{0}^{3} c_{n n v}+S_{0} b_{E E}, \\
& S_{N N E}=S_{0}^{3} c_{n n e}
\end{aligned}
$$

with

$$
\begin{aligned}
& S_{V V N}=-S_{0}^{3} c_{v v n}-2 S_{0} b_{1 E^{\prime}} \beta E_{1} \\
& S_{V V V}=S_{0}^{3} c_{v v v}+3 S_{0} b_{E^{\prime} E^{\prime}} \\
& S_{V V E}=S_{0}^{3} c_{v v e}+2 S_{0} b_{1 E^{\prime}}
\end{aligned}
$$

and

and finally

$$
\begin{aligned}
& S_{E E N}=-S_{0}^{3} c_{e e n}, \\
& S_{E E V}=S_{0}^{3} c_{e e v}+S_{0} b_{11}, \\
& S_{E E E}=S_{0}^{3} c_{e e e}
\end{aligned}
$$

$$
S_{N V E}=-S_{0}^{3} c_{n v e}-S_{0} b_{1 E},
$$

where we have used the following definitions:

$$
\begin{aligned}
& n \equiv \beta E b_{1 E}-N_{1} b_{E E}, \\
& v \equiv \beta E^{\prime} b_{1 E^{\prime}}-N_{1} b_{E^{\prime} E^{\prime}}, \\
& e \equiv \beta E b_{11}-N_{1} b_{1 E} .
\end{aligned}
$$

While most of these derivatives are available experimentally, the particle number derivatives are not due to their dependence 
on the zero of energy. ${ }^{24}$ Note that, in the above volume derivatives of the entropy, there is an additional term involving pair fluctuations that will only contribute for isobaric ensembles.

\section{RESULTS AND DISCUSSION}

\section{A. Gaussian distributions}

Gaussian approximations for the particle, energy, and volume distributions are common in textbooks. ${ }^{2,16}$ However, we have included them here for three main reasons. First, they illustrate the process of obtaining the distributions from the second entropy derivatives, which is then extended to include the third derivatives in Sec. III B. Second, the forms that most commonly appear in textbooks often treat multiple fluctuating variables independently, whereas in reality they are usually correlated. In many cases, this limitation is not a major problem as only the order of magnitude of the fluctuation is of interest. However, the appropriate multivariate Gaussian distribution can also be of importance. Third, an initial analysis of the $N V T, N p T$, and $\mu V T$ results suggested that a single probability distribution valid for a variety of ensembles is available.

As mentioned previously, many of the entropy derivatives are not available experimentally. This indicates that some form of substitution is required to provide combinations of entropy derivatives that are experimentally available. Based on our previous applications of FST, ${ }^{19}$ we will adopt the following dimensionless quantities:

$$
\begin{aligned}
V^{E} & \equiv \rho_{1}\left(V-N_{1} V_{1}\right), \\
H^{E} & \equiv \beta\left(E+\langle p\rangle V-N_{1} H_{1}\right), \\
S^{E} & \equiv\left(S-N_{1} S_{1}\right) / R
\end{aligned}
$$

representing an instantaneous excess volume, enthalpy, and entropy, respectively. Here, $S_{1}$ is the molar entropy and the values of $N_{1}, V, E$, and $S$ can fluctuate depending on the ensemble. These excess quantities average to zero in all the main ensembles. The enthalpy involves the average pressure written as $\langle p\rangle$ to emphasize that pressure never fluctuates in these quantities regardless of the ensemble. Furthermore, the excess enthalpy definition avoids the zero of energy problem for open systems. ${ }^{24}$ Before using the above quantities to develop the entropy expansion, we note that the corresponding quantities, $\rho_{1} \delta V=\delta V^{E}+\delta N_{1}$ and $\beta \delta E=\delta H^{E}-Z \delta V^{E}+\beta E_{1} \delta N_{1}$, when used in Equation (6) give

$$
\delta S^{E}=\delta H^{E}
$$

for small fluctuations. Hence, the excess entropy and enthalpy fluctuations are equal and therefore $\delta S^{E}$ could be used in place of $\delta H^{E}$ in the following equations.

If we substitute the expressions for $\delta E$ and $\delta V$ given by Equation (20) into the expression for $S^{(2)}$ found in Equation (5) and then collect identical terms the result can be written as

$$
\begin{aligned}
S^{(2)}= & F_{V V}\left(\delta V^{E}\right)^{2}+F_{H H}\left(\delta H^{E}\right)^{2}+F_{N N}\left(\delta N_{1}\right)^{2} \\
& +2 F_{V H} \delta V^{E} \delta H^{E}+2 F_{N V} \delta N_{1} \delta V^{E}+2 F_{N H} \delta N_{1} \delta H^{E},
\end{aligned}
$$

where the $F$ 's involve combinations of second entropy derivatives. Interestingly, the $F$ 's corresponding to the particle number fluctuations are given by

$$
\begin{aligned}
F_{N N} \equiv & S_{N N}+S_{V V}+S_{E E}\left(\beta E_{1}\right)^{2}+2 S_{N V}+2 S_{N E} \beta E_{1} \\
& +2 S_{V E} \beta E_{1}=0 \\
F_{N V} \equiv & S_{V V}-S_{E E} \beta E_{1} Z+S_{N V}-S_{N E} Z \\
& +S_{V E}\left(\beta E_{1}-Z\right)=0 \\
F_{N H} \equiv & S_{E E} \beta E_{1}+S_{N E}+2 S_{V E}=0
\end{aligned}
$$

and subsequently disappear. The other $F$ 's simplify considerably to give

$$
\begin{aligned}
& F_{V V} \equiv S_{V V}-2 S_{V E} Z+S_{E E} Z^{2}=-S_{0} b_{\varepsilon \varepsilon} \\
& F_{V H} \equiv S_{V E}-S_{E E} Z=-S_{0} b_{1 \varepsilon} \\
& F_{H H} \equiv S_{E E}=-S_{0} b_{11}
\end{aligned}
$$

all of which involve quantities that are available experimentally. Therefore, the FST based Gaussian probability distribution is then

$$
\begin{aligned}
\ln P\left(N_{1}, V, E\right)= & -\frac{S_{0}}{2 N_{1}}\left[b_{\varepsilon \varepsilon}\left(\delta V^{E}\right)^{2}+2 b_{1 \varepsilon} \delta V^{E} \delta H^{E}\right. \\
& \left.+b_{11}\left(\delta H^{E}\right)^{2}\right]
\end{aligned}
$$

which can be expressed using common thermodynamic properties as

$$
\begin{aligned}
\ln P\left(N_{1}, V, E\right)= & -\frac{1}{2 N_{1}} \frac{C_{p, m}}{C_{V, m}}\left[\frac{\left(\delta V^{E}\right)^{2}}{p \kappa_{T} / Z}\right. \\
& \left.-2 T \alpha_{p} \frac{\delta V^{E}}{p \kappa_{T} / Z} \frac{\delta H^{E}}{C_{p, m} / R}+\frac{\left(\delta H^{E}\right)^{2}}{C_{p, m} / R}\right] .
\end{aligned}
$$

The relationship is valid for all the common ensembles of interest. Specific expressions for each ensemble are provided below.

The normalized Gaussian probability distribution result for ensembles where at least one extensive variable is fixed, i.e., all but the $\mu p T$ ensemble, can be written in a general form given by

$$
P\left(N_{1}, V, E\right)=\left(2 \pi|\mathbf{Q}|^{1 / 2}\right)^{-1} e^{-\frac{1}{2} \mathbf{q}^{\mathrm{T}} \mathbf{Q}^{-1} \mathbf{q},}
$$

where the covariance matrix, $\mathbf{Q}$, involves the pair fluctuations arising from a FST analysis of the thermodynamics according to

$$
\mathbf{Q} \equiv\left(\begin{array}{cc}
\left\langle\delta N_{1} \delta N_{1}\right\rangle & -\beta\left\langle\delta N_{1} \delta \varepsilon\right\rangle \\
-\beta\left\langle\delta N_{1} \delta \varepsilon\right\rangle & \beta^{2}\langle\delta \varepsilon \delta \varepsilon\rangle
\end{array}\right) .
$$

Alternatively, in terms of common thermodynamic properties one has

$$
\mathbf{Q}=N_{1}\left(\begin{array}{cc}
p \kappa_{T} / Z & T \alpha_{p} \\
T \alpha_{p} & C_{p, m} / R
\end{array}\right),
$$

where $|\mathbf{Q}|=C_{V} p \kappa_{T} /(R Z)=N_{1} / S_{0}$. The thermodynamic properties in the above matrix provide the covariances corresponding to the fluctuations in the excess volume and enthalpy as indicated in Equation (26). The general nature of the above distribution is then provided by the corresponding column vector which takes the form

$$
\mathbf{q} \equiv\left(\begin{array}{c}
\delta V^{E} \\
\delta H^{E}
\end{array}\right)
$$

in terms of the excess volume and enthalpy. This then reduces to the following values for the main ensembles: 


$$
\begin{aligned}
\mathbf{q} & =\left(\begin{array}{l}
0 \\
0
\end{array}\right)_{N V E}=\left(\begin{array}{c}
0 \\
\beta \delta E
\end{array}\right)_{N V T}=\left(\begin{array}{c}
\rho_{1} \delta V \\
\beta \delta H
\end{array}\right)_{N p T} \\
& =\left(\begin{array}{c}
-\delta N_{1} \\
\beta \delta \varepsilon
\end{array}\right)_{\mu V T}=\left(\begin{array}{c}
\rho_{1} \delta V \\
0
\end{array}\right)_{N p H}
\end{aligned}
$$

although the $\mathrm{NpH}$ ensemble is usually only of significant interest for computer simulation studies. ${ }^{28}$

Consequently, a Gaussian result valid for all ensembles has been provided in terms of an excess volume and enthalpy. The excess volume and enthalpy then reduce to the appropriate fluctuating quantities for each ensemble, while the covariance matrix itself does not change. The fluctuating quantities are essentially those expected for all but the GCE. In this latter case, the above relationships clearly indicate that the excess energy $\varepsilon$ is the relevant quantity in the GCE ensemble required to describe the common thermodynamic properties in the simplest manner. The above equations also emphasize the cross correlation between the fluctuations in the $N p T$ and GCE that only disappears when $\alpha_{p}=0$ and hence $C_{p, m}=C_{V, m}$. Finally, we note that the determinant of the $3 \times 3$ matrix of entropy derivatives formed from the relationships provided in Equation (14) can be shown to be singular, and hence a general multivariate Gaussian distribution function expression for the (unbounded) fluctuations cannot be written for the corresponding $\mu p T$ ensemble (as expected).

\section{B. Non-Gaussian distribution}

The corresponding non-Gaussian distribution is significantly more complicated. In particular, while the number of fluctuating quantities remains reasonable, the number of corresponding thermodynamic derivatives increases substantially. Nevertheless, from the previous results, it would appear that a general distribution valid for multiple ensembles should be available. Again, we use the substitutions denoted in Equation (20) for the $S^{(2)}$ and $S^{(3)}$ terms. The $S^{(2)}$ term remains the same as before and we can use the result presented in Equation (25) or (26). We will factor the $S^{(3)}$ term into two contributions, one $\left(P_{V}\right)$ involving just the pair fluctuations ( $b$ 's) that only appears for isobaric ensembles, and one $\left(P_{3}\right)$ involving just the triplet fluctuations ( $c$ 's), as indicated in Equations (15)-(18). The same type of manipulations used in Sec. III A is required again. The details are provided in Appendix D.

This approach leads to a general expression for the distribution

$$
\ln P\left(N_{1}, V, E\right)=P_{2}+P_{V}+P_{3},
$$

where

$$
\begin{aligned}
P_{2}= & -\frac{S_{0}}{2 N_{1}}\left[b_{\varepsilon \varepsilon}\left(\delta V^{E}\right)^{2}+2 b_{1 \varepsilon} \delta V^{E} \delta H^{E}+b_{11}\left(\delta H^{E}\right)^{2}\right] \\
P_{V}= & \frac{S_{0}}{2 N_{1}^{2}}\left[b_{\varepsilon \varepsilon}\left(\delta V^{E}\right)^{3}+2 b_{1 \varepsilon}\left(\delta V^{E}\right)^{2} \delta H^{E}+b_{11} \delta V^{E}\left(\delta H^{E}\right)^{2}\right] \\
= & -P_{2} \delta V^{E} / N_{1}, \\
P_{3}= & \frac{S_{0}^{3}}{6 N_{1}^{2}}\left[c_{s s s}\left(\delta V^{E}\right)^{3}+3 c_{s s t}\left(\delta V^{E}\right)^{2} \delta H^{E}+3 c_{s t t} \delta V^{E}\left(\delta H^{E}\right)^{2}\right. \\
& \left.+c_{t t t}\left(\delta H^{E}\right)^{3}\right]
\end{aligned}
$$

in terms of the fluctuations defined in Equation (20) and where

$$
\begin{aligned}
s & \equiv \beta \varepsilon b_{1 \varepsilon}-N_{1} b_{\varepsilon \varepsilon}, \\
t & \equiv \beta \varepsilon b_{11}-N_{1} b_{1 \varepsilon} .
\end{aligned}
$$

We note that the $P_{V}$ term only contributes for isobaric ensembles (even if the particle number or energy fluctuates). The intensive properties defined in Equation (8) indicate that the pair and triplet fluctuations are proportional to $V$ and therefore $N_{1}$. Consequently, the $P_{V}$ and $P_{3}$ terms are not intensive and appear to become negligible as the system size becomes infinite. Hence, the real distribution then tends towards that of a Gaussian distribution. This is as expected, although the infinite system limit is complicated by the additional requirement of an infinite "bath" to maintain a constant chemical potential, temperature, or pressure. ${ }^{11}$ However, as we show below, this does not mean that the third and higher moments, obtained upon integration, are then zero.

$P_{2}$ and $P_{\mathrm{V}}$ can be expressed in terms of thermodynamic properties using the results given in Equation (11). In order to express the above result for $P_{3}$ in terms of thermodynamic properties, we need to express the triplet fluctuations in terms of thermodynamic properties. This process is described in Appendix D. The final result is

$$
\begin{aligned}
& S_{0}^{3} c_{t t t}=\left(\frac{R}{C_{V, m}}\right)^{2} J_{1}, \\
& S_{0}^{3} c_{s t t}=-\left(\frac{R}{C_{V, m}}\right)^{2}\left[\left(Z \frac{T}{p} \frac{\partial p}{\partial T}\right)_{\rho_{1}} J_{1}+\frac{C_{V, m}}{R} J_{2}\right], \\
& S_{0}^{3} c_{s s t}=\left(\frac{R}{C_{V, m}}\right)^{2}\left[\left(Z \frac{T}{p} \frac{\partial p}{\partial T}\right)_{\rho_{1}}^{2} J_{1}+2 \frac{C_{V, m}}{R}\left(Z \frac{T}{p} \frac{\partial p}{\partial T}\right)_{\rho_{1}} J_{2}\right. \\
& \left.+\frac{C_{V, m}}{R} \frac{Z}{p \kappa_{T}} J_{3}\right] \\
& S_{0}^{3} c_{s s s}=-\left(\frac{R}{C_{V, m}}\right)^{2} \\
& \times\left[\begin{array}{c}
\left(Z \frac{T}{p} \frac{\partial p}{\partial T}\right)_{\rho_{1}}^{3} J_{1}+3 \frac{C_{V, m}}{R}\left(Z \frac{T}{p} \frac{\partial p}{\partial T}\right)_{\rho_{1}}^{2} J_{2} \\
+3 \frac{C_{V, m}}{R} \frac{Z}{p \kappa_{T}}\left(Z \frac{T}{p} \frac{\partial p}{\partial T}\right)_{\rho_{1}} J_{3}+\left(\frac{C_{V, m}}{R}\right)^{2} \frac{Z}{p \kappa_{T}} J_{4}
\end{array}\right],
\end{aligned}
$$

where the $J_{\mathrm{n}}$ 's are given by

$$
\begin{aligned}
& J_{1} \equiv \frac{c_{t t t}}{b_{11} b_{t t}}=2+\frac{T}{C_{V, m}}\left(\frac{\partial C_{V, m}}{\partial T}\right)_{\rho_{1}}, \\
& J_{2} \equiv \frac{c_{1 t t}}{b_{11} b_{t t}}=1-Z \frac{R}{C_{V, m}} \frac{T^{2}}{p}\left(\frac{\partial^{2} p}{\partial T^{2}}\right)_{\rho_{1}}, \\
& J_{3} \equiv \frac{c_{11 t}}{b_{11}^{2}}=1+\frac{T}{\kappa_{T}}\left(\frac{\partial \kappa_{T}}{\partial T}\right)_{\rho_{1}}, \\
& J_{4} \equiv \frac{c_{111}}{b_{11}^{2}}=1+\rho_{1}\left(\frac{\partial^{2} \rho_{1}}{\partial p^{2}}\right)_{T} /\left(\frac{\partial \rho_{1}}{\partial p}\right)_{T}^{2}
\end{aligned}
$$

and have been written so that the distribution is Gaussian when all the $J_{\mathrm{n}}$ 's are zero. Furthermore, the coefficients of the $J_{\mathrm{n}}$ 's 
are always positive, in the absence of an isobaric density maximum, and therefore the $J_{\mathrm{n}}$ 's also determine the sign of the $c$ 's. The various combinations of derivatives in Equation (39) characterize the deviation from Gaussian behavior. The distribution provided by Equations (32), (33), and (35)-(39) is valid for all the ensembles described in Section III A. When $\alpha_{p}$ is zero then so is the thermal pressure coefficient. Under these conditions, the expressions simplify greatly as only the final term on the right hand side of Equations (35)-(38) survives. The $J_{\mathrm{n}}$ 's are clearly related to the GCE triplet fluctuations and hence finite triplet fluctuations lead to non-Gaussian behavior. This is to be expected, but now it is clear which triplet fluctuations are important and how they relate to more common thermodynamic derivatives. Finally, the value of $J_{3}$ is given by a thermodynamic derivative not commonly provided by a typical equation of state. An alternative expression that employs more traditional derivatives is given by

$J_{3}=1+T\left[\left(\frac{\partial^{2} \rho_{1}}{\partial p \partial T}\right)\left(\frac{\partial \rho_{1}}{\partial p}\right)_{T}-\left(\frac{\partial^{2} \rho_{1}}{\partial p^{2}}\right)_{T}\left(\frac{\partial \rho_{1}}{\partial T}\right)_{p}\right] /\left(\frac{\partial \rho_{1}}{\partial p}\right)_{T}^{2}$

and was obtained using the relationships provided in Equation (D5).

\section{Experimental non-Gaussian behavior}

To investigate the non-Gaussian behavior further, we have extracted the $J_{n}$ 's from accurate equations of state for the complex fluid water, ${ }^{29}$ and the simple fluid argon, ${ }^{30}$ that provide the required thermodynamic derivatives. The results are presented in Figures 1 and 2 and also in Tables I and II. It is immediately apparent that there is no point on the phase diagrams where all four $J_{\mathrm{n}}$ 's are zero. While several of the $J_{\mathrm{n}}$ 's do change sign, particularly in the liquid and supercritical regions, they do so at different pressures and temperatures. Non-Gaussian behavior can be quantified by the $J_{\mathrm{n}}$ values. When the $J_{\mathrm{n}}$ 's are all zero, one observes a simple Gaussian distribution. This places firm restrictions on the allowed values of the thermodynamic derivatives in Equation (39). The data for water presented in Table II clearly show that the distribution displays significant non-Gaussian character. Hence, these restrictions are unlikely to be met for real fluids at a single state point and Gaussian behavior in pure fluids does not appear to be possible. The ideal gas results are also included in Table I to emphasize that Gaussian behavior is also not observed even in this limiting case. In fact, ideal gases actually follow a Poisson distribution. ${ }^{9}, 14$ It should be noted that the $J_{\mathrm{n}}$ 's and $c$ 's in Table I represent triplet fluctuations in the GCE. As these are

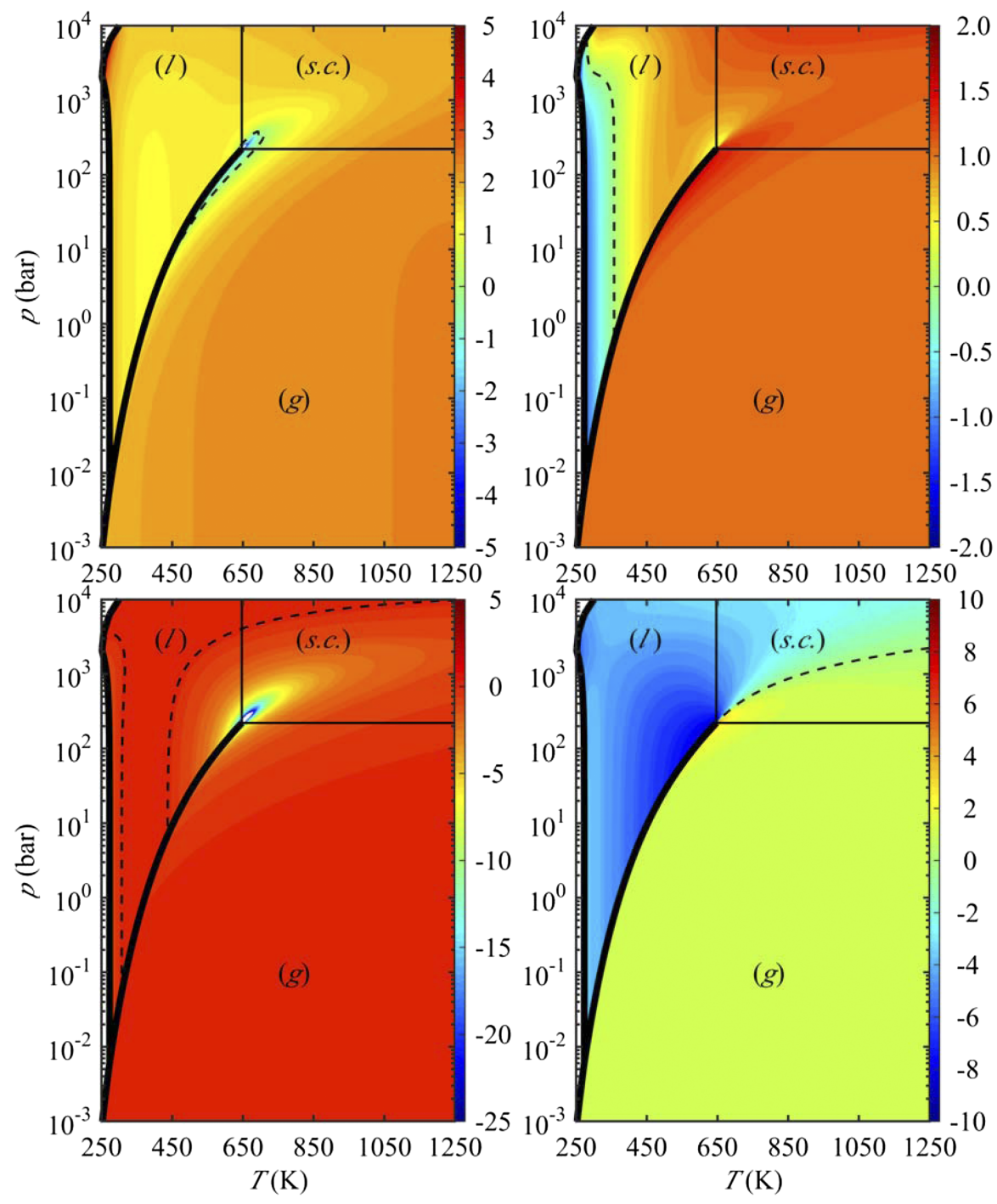

FIG. 1. Contour plots for the dimensionless non-Gaussian measures $J_{\mathrm{n}}$ as a function of $p$ and $T$ for fluid water. Top: $J_{1}$ (left) and $J_{2}$ (right). Bottom: $J_{3}$ (left) and $J_{4}$ (right). The thick solid black curves denote the phase boundaries. Zero contours are indicated with dashed black curves. The boundary of the supercritical region is marked by thin black lines. The data were generated using the IAPWS-95 equation of state, ${ }^{29}$ as implemented in the NIST/ASME STEAM database version $2.2 .^{31}$ 

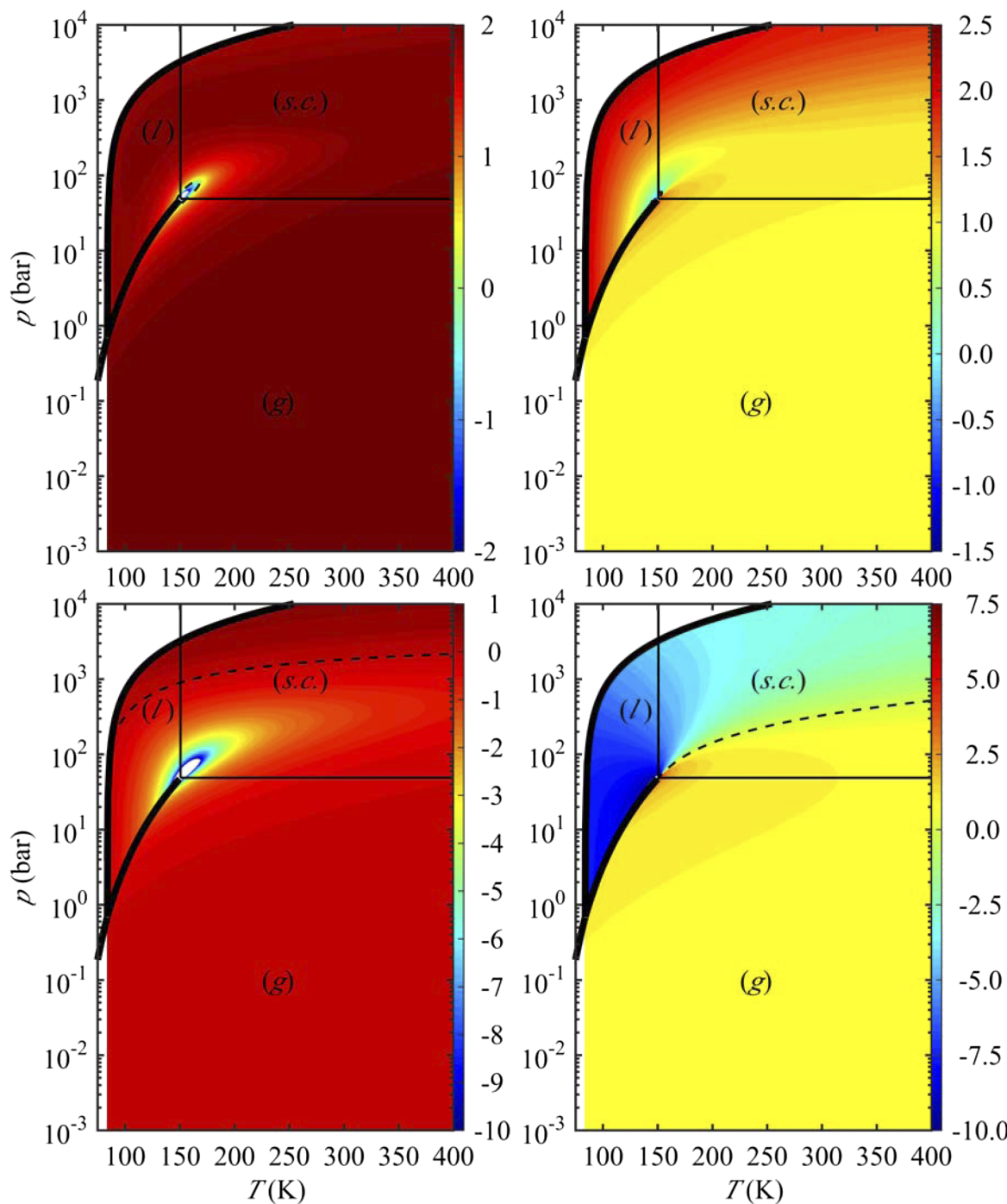

FIG. 2. Contour plots for the dimensionless non-Gaussian measures $J_{\mathrm{n}}$ as a function of $p$ and $T$ for fluid argon. Top: $J_{1}$ (left) and $J_{2}$ (right). Bottom: $J_{3}$ (left) and $J_{4}$ (right). The thick solid black curves denote the phase boundaries. Zero contours are indicated with dashed black curves. The boundary of the supercritical region is marked by thin black lines. The data were generated using the equation of state from Tegeler, Span, and Wagner, ${ }^{30}$ as implemented in the NIST REFPROP database version $9.1 .^{32}$ available and finite, this indicates that the third moments of the distribution, i.e., the measures of skewness, are non-zero for real fluids. Consequently, although the probability distribution may visually tend towards a Gaussian distribution for infinite systems, the resulting third moments obtained on integration are still finite-assuming that real fluid behavior represents the true thermodynamic limit.

\section{Specific distributions for the common ensembles}

The general result given in Equation (33) can be used to provide specific expressions for different ensembles. For the canonical ensemble we find

$$
\begin{aligned}
\ln P\left(E \mid N_{1}, V\right)= & -\frac{S_{0}}{2 N_{1}}\left[b_{11}(\beta \delta E)^{2}\right] \\
& +\frac{S_{0}^{3}}{6 N_{1}^{2}}\left[c_{t t t}(\beta \delta E)^{3}\right] .
\end{aligned}
$$

From Equation (21) or, equivalently, Equation (6), we also have $\delta S / R=\beta \delta E$ or $\delta A=0$ for (infinitesimally) small fluctuations. Clearly, the Gaussian result corresponds to truncation after the first term on the right-hand side. The result can also be expressed in terms of common thermodynamic properties using the previous relationships.
For the isothermal-isobaric ensemble we have

$$
\begin{aligned}
\ln P\left(V, E \mid N_{1}\right)= & -\frac{S_{0}}{2 N_{1}}\left[b_{\varepsilon \varepsilon}\left(\rho_{1} \delta V\right)^{2}+2 b_{1 \varepsilon} \rho_{1} \delta V \beta \delta H\right. \\
& \left.+b_{11}(\beta \delta H)^{2}\right]\left(1-\frac{\rho_{1} \delta V}{N_{1}}\right) \\
& +\frac{S_{0}^{3}}{6 N_{1}^{2}}\left[c_{s s s}\left(\rho_{1} \delta V\right)^{3}+3 c_{s s t}\left(\rho_{1} \delta V\right)^{2} \beta \delta H\right. \\
& \left.+3 c_{s t t} \rho_{1} \delta V(\beta \delta H)^{2}+c_{t t t}(\beta \delta H)^{3}\right]
\end{aligned}
$$

and $\delta S / R=\beta \delta H$ or $\delta G=0$. One could expand the enthalpy to give the energy and volume fluctuations. However, this does not provide the simplest relationships for the corresponding moments.

In the GCE we have

$$
\begin{aligned}
\ln P\left(N_{1}, E \mid V\right)= & -\frac{S_{0}}{2\left\langle N_{1}\right\rangle}\left[b_{\varepsilon \varepsilon}\left(\delta N_{1}\right)^{2}-2 b_{1 \varepsilon} \delta N_{1} \beta \delta \varepsilon\right. \\
& \left.+b_{11}(\beta \delta \varepsilon)^{2}\right] \\
& -\frac{S_{0}^{3}}{6\left\langle N_{1}\right\rangle^{2}}\left[c_{s s s}\left(\delta N_{1}\right)^{3}-3 c_{s s t}\left(\delta N_{1}\right)^{2} \beta \delta \varepsilon\right. \\
& \left.+3 c_{s t t} \delta N_{1}(\beta \delta \varepsilon)^{2}-c_{t t t}(\beta \delta \varepsilon)^{3}\right]
\end{aligned}
$$


TABLE I. Signs for the non-Gaussian measures in different fluid phases of water and argon. S.C. $=$ supercritical; I.G. = classical ideal gas; $C_{r}=C_{V, m} / R$, where the heat capacity corresponds to that provided by the translational and intramolecular rotational and vibrational degrees of freedom, which are considered independent of temperature.

\begin{tabular}{lcccccccc}
\hline \hline & \multicolumn{5}{c}{ Water } & & \multicolumn{3}{c}{ Argon } & \\
\cline { 2 - 4 } Property & Gas & Liquid & S.C. & Gas & Liquid & S.C. & I.G. \\
\hline$S_{0}$ & + & + & + & + & + & + & $1 / C_{r}$ \\
$b_{11}$ & + & + & + & + & + & + & 1 \\
$b_{1 \varepsilon} \mathrm{a}$ & - & - & - & - & - & - & -1 \\
$b_{\varepsilon \varepsilon}$ & + & + & + & + & + & + & $1+C_{r}$ \\
$J_{1}$ & + & + & $+/-$ & + & + & $+/-$ & 2 \\
$J_{2}$ & + & $+/-$ & + & + & $+/-$ & + & 1 \\
$J_{3}$ & - & $+/-$ & $+/-$ & - & - & - & 0 \\
$J_{4}$ & + & - & $+/-$ & + & - & $+/-$ & 1 \\
$c_{t t t}$ & + & + & $+/-$ & + & + & $+/-$ & $2 C_{r}$ \\
$c_{s t t}$ & - & $+/-$ & - & - & $+/-$ & $+/-$ & $-C_{r}\left(2+C_{r}\right)$ \\
$c_{s s t}$ & + & $+/-$ & + & + & $+/-$ & $+/-$ & $2 C_{r}\left(1+C_{r}\right)$ \\
$c_{s s s}$ & - & + & $+/-$ & - & $+/-$ & $+/-$ & $-C_{r}\left(1+C_{r}\right)\left(2+C_{r}\right)$ \\
\hline \hline
\end{tabular}

${ }^{\mathrm{a}}$ The value of $\mathrm{b}_{1 \varepsilon}$ can be positive, negative, or zero (for liquid water) but is negative for the vast majority of the phase diagram. We have ignored changes in sign close to the vapor-liquid curve and the critical point.

and $\delta S^{E} / R=\beta \delta \varepsilon$ or $\delta A-\mu_{1} \delta N_{1}=0$. Hence, the excess energy is equivalent to the excess entropy for this ensemble. This condition can be rewritten to include the thermodynamic potential associated with the GCE to give $\delta G-\mu_{1} \delta N_{1}-V \delta p$ $=0$. But, $\delta G=\mu_{1} \delta N_{1}$ for the GCE, and so we have $\delta p=0$. Again, one can expand the excess energy term but, not only do the expressions become more complicated, they also lead to energy moments that are experimentally inaccessible.

Finally, the isobaric-isenthalpic ensemble leads to

$$
\begin{aligned}
\ln P\left(V \mid N_{1}, H\right)= & -\frac{S_{0}}{2 N_{1}}\left[b_{\varepsilon \varepsilon}\left(\rho_{1} \delta V\right)^{2}\right]\left(1-\frac{\rho_{1} \delta V}{N_{1}}\right) \\
& +\frac{S_{0}^{3}}{6 N_{1}^{2}}\left[c_{s s s}\left(\rho_{1} \delta V\right)^{3}\right]
\end{aligned}
$$

and $\delta S=0$. All the above equations can be expressed in terms of common thermodynamic properties using Equations (10) and (35)-(38).

\section{E. Properties of the distributions}

Previously we stated that, while the distribution tends towards a Gaussian for large systems, the higher moments (coskewness, excess cokurtosis) of the distribution are still finite. Here, we illustrate this behavior in more detail. Unfortunately, the entropy expansion through $S^{(3)}$ results in a distribution that cannot be integrated and hence the moments are unavailable. To provide a distribution that can be integrated, one requires higher terms in the expansion. As the general

TABLE II. Values for the non-Gaussian measures in liquid water at $298.15 \mathrm{~K}$ and 1 bar.

\begin{tabular}{lrlrlr}
\hline \hline$S_{0}$ & 1.797 & $J_{1}$ & 1.577 & $c_{t t t}$ & 0.003 \\
$b_{11}$ & 0.062 & $J_{2}$ & -0.800 & $c_{s t t}$ & 0.011 \\
$b_{1 \varepsilon}$ & -0.077 & $J_{3}$ & -0.170 & $c_{s s t}$ & -0.085 \\
$b_{\varepsilon \varepsilon}$ & 9.060 & $J_{4}$ & -3.682 & $c_{s s s}$ & 10.484 \\
\hline \hline
\end{tabular}

expression for the fluctuations leads to many complicated additional terms, we have focused on the simplest case- the energy distribution in the canonical ensemble — where we only require one extra derivative. This process is outlined in Appendix E. In particular, we compare the moments obtained from the current entropy expansion to the known (exact) moments obtained from the canonical ensemble partition function to ensure that the results are consistent.

Before using the expanded energy distribution, we note that the exact central moments $\left(M_{\mathrm{n}}\right)$ of the canonical ensemble energy distribution can be obtained by repeated differentiation of the partition function with respect to temperature. ${ }^{2}$ This also provides the exact cumulants $\left(K_{\mathrm{n}}\right)$ of the distribution in terms of the central moments. The results are

$$
\begin{gathered}
\langle\beta \delta E\rangle=M_{1}=0, \\
\frac{C_{V}}{R}=\left\langle(\beta \delta E)^{2}\right\rangle=M_{2}=K_{2}=\sigma^{2}, \\
2 \frac{C_{V}}{R}+\frac{T}{R}\left(\frac{\partial C_{V}}{\partial T}\right)_{\rho_{1}}=\left\langle(\beta \delta E)^{3}\right\rangle=M_{3}=K_{3}, \\
6 \frac{C_{V}}{R}+6 \frac{T}{R}\left(\frac{\partial C_{V}}{\partial T}\right)_{\rho_{1}}+\frac{T^{2}}{R}\left(\frac{\partial^{2} C_{V}}{\partial T^{2}}\right)_{\rho_{1}} \\
=\left\langle(\beta \delta E)^{4}\right\rangle-3\left\langle(\beta \delta E)^{2}\right\rangle^{2}=M_{4}-3 M_{2}^{2}=K_{4},
\end{gathered}
$$

where $\sigma^{2}$ is the variance of the energy pdf and all the ensemble averages correspond to the canonical ensemble. The above expressions can be used to relate the fluctuations in the GCE and canonical ensembles as indicated in Equations (D4), (D6), and (E3).

All the pdfs presented in Secs. III A and III D actually depend on the system size. However, in most cases, one is only interested in the thermodynamic limit, i.e., $N_{1} \rightarrow \infty$ with $\rho_{1}$ fixed. It is commonly noted that in the thermodynamic limit, the most probable and the average quantities coincide. However, while this is true in the Gaussian approximation, this is not true for the non-Gaussian counterparts. Hence, the equations presented in Sections III B and III D do not provide $\langle E\rangle=E^{*}$, etc. Clearly, for a finite system, we actually have $\delta E$ $=E-E^{*}$ as the pdfs display a maximum at $\delta E=0$. This does not invalidate the pdfs described previously, but it does have some subtle, and important, consequences that are discussed below.

Using the results presented in Equations (14), (17), (39), (E1), (E3), and (45) in the entropy expansion, we find that

$$
\begin{aligned}
\ln P\left(E \mid N_{1}, V\right)= & -\frac{K_{2}}{2}\left(\frac{\beta \delta E}{K_{2}}\right)^{2}\left[1-\frac{1}{3} \frac{K_{3}}{K_{2}}\left(\frac{\beta \delta E}{K_{2}}\right)\right. \\
& \left.-\frac{1}{12}\left(\frac{K_{4}}{K_{2}}-3 \frac{K_{3}^{2}}{K_{2}^{2}}\right)\left(\frac{\beta \delta E}{K_{2}}\right)^{2}\right] .
\end{aligned}
$$

This is a general expression for the energy fluctuations in the canonical ensemble through fourth order. It is interesting to compare the central moments $\left(\tilde{M}_{n}\right)$ and corresponding cumulants $\left(\tilde{K}_{n}\right)$ of the distribution provided by Equation (46) with the known exact central moments and cumulants given in Equation (45). First, we note that the moments provided by Equation (46) depend on $N_{1}$, as does the visual appearance 


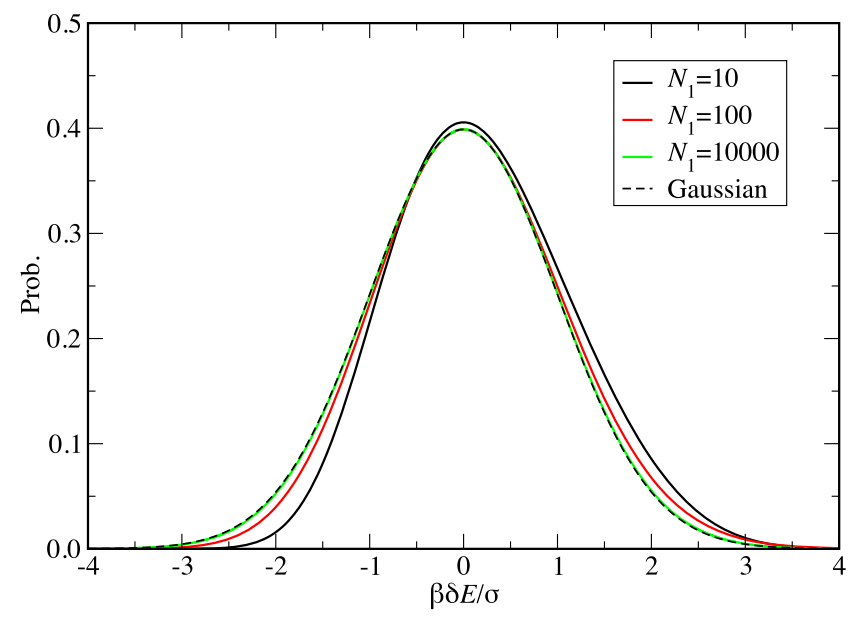

FIG. 3. Energy fluctuation probability distributions for the canonical ensemble as provided by Equation (46) in the high temperature limit. The normalized curves correspond to different systems sizes where $C_{V, m} / R=1$, but the $C_{V} / R=N_{1}=K_{2}=\sigma^{2}$ values differ. The moments and cumulants obtained for infinite size systems correspond to exact values of $M_{1} / K_{2}=0, K_{3} / K_{2}=2$, and $K_{4} / K_{2}=6$.

of the distribution. This is illustrated in Figure 3 where we present the energy pdfs for different system sizes and compare the results with the corresponding Gaussian distribution. While the skewness and kurtosis are apparent for small system sizes, this appears to vanish for large systems and the distribution then visually resembles a Gaussian. This is due to the fact that the skewness and kurtosis become small in comparison with the variance. Numerical integration of the pdf for an infinite system provides

$$
\begin{array}{lll}
\frac{\tilde{M}_{1}}{\tilde{M}_{2}^{1 / 2}}=0, & & \frac{\tilde{M}_{3}}{\tilde{M}_{2}^{3 / 2}}=0, \\
\frac{\tilde{M}_{4}}{\tilde{M}_{2}^{2}}=3, & \frac{\tilde{K}_{4}}{\tilde{M}_{2}^{2}}=0
\end{array}
$$

for the standardized moments, as would be expected for a Gaussian distribution.

Upon integration, however, the asymmetry in the distribution is magnified and gives rise to non-zero values for the third and fourth cumulants. In fact, by numerical integration of Equation (46), we find that

$$
\begin{aligned}
& \beta\left(\langle E\rangle-E^{*}\right)=\frac{1}{2} \frac{K_{3}}{K_{2}}, \\
& \frac{\tilde{K}_{2}}{K_{2}}=\frac{\tilde{K}_{3}}{K_{3}}=\frac{\tilde{K}_{4}}{K_{4}}=1
\end{aligned}
$$

for $N_{1} \rightarrow \infty$. The relationships are also good for $N_{1}>\approx 1000$. Hence, the cumulants remain finite even though the distribution appears Gaussian. It is the cumulant ratios that give rise to the associated thermodynamic behavior, i.e., the changes in thermodynamic properties with changes in state point, as shown in Equation (45). For an infinite system, one obtains the same central moments and cumulants as provided by differentiation of the canonical ensemble partition function. The above results suggest that an analytical expression for the moments of the distribution should be available. However, we were unable to find one. The raw moments and corresponding cumulants $\left(\hat{K}_{n}\right)$ are different from the central moments, as indicated by the first relationship in Equation (48), and are given by

$$
\frac{\hat{K}_{2}}{K_{2}}=1, \quad \frac{\hat{K}_{3}}{K_{2}}=\frac{5}{2} \frac{K_{3}}{K_{2}}, \quad \frac{\hat{K}_{4}}{K_{2}}=\frac{K_{4}}{K_{2}}+2\left(\frac{K_{3}}{K_{2}}\right)^{2}
$$

for the distribution in Equation (46) in the infinite system limit.

As mentioned previously, if the $(\delta E)^{4}$ term in Equation (46) is set to zero, then the pdf cannot be (formally) integrated due to the behavior of the pdf as $\delta E \rightarrow \infty$. However, our numerical studies indicate that one actually finds a wellbehaved Gaussian-like probability distribution followed by a divergence for energy fluctuations well removed from the main $(\delta E \approx 0)$ distribution. The appearance of the divergence shifts to higher and higher energy fluctuations as $N_{1}$ increases (roughly as $N_{1} \sigma$ ). Consequently, for reasonably large values of $N_{1}$, the energy pdf is perfectly well behaved as long as one truncates the integration at $10 \sigma$ or so. In this case, the same results as described in Equation (48) are obtained.

The above relationships for the cumulants in terms of thermodynamic derivatives contain many terms. However, if we ignore the temperature dependence of the heat capacity, which is a valid approximation at high temperatures, then the fluctuations and entropy derivatives are simply given by the first term on the left hand side of Equation (45). The pdf can then be written as

$$
\begin{aligned}
\ln P\left(E \mid N_{1}, V\right)= & \frac{C_{V}}{R}\left[-\frac{1}{2}\left(\frac{\beta \delta E}{C_{V} / R}\right)^{2}+\frac{1}{3}\left(\frac{\beta \delta E}{C_{V} / R}\right)^{3}\right. \\
& \left.-\frac{1}{4}\left(\frac{\beta \delta E}{C_{V} / R}\right)^{4}\right]
\end{aligned}
$$

which takes the form of a $\ln (1+x)$ expansion. Assuming this is also true beyond fourth order, we find that for small $\left(|\beta \delta E|<C_{V} / R\right)$ energy fluctuations, the un-normalized energy pdf is given by

$$
P\left(E \mid N_{1}, V\right)=\left(1+\frac{\beta \delta E}{C_{V} / R}\right)^{C_{V} / R} e^{-\beta \delta E}
$$

through all orders, and also provides the entropy fluctuations via Equation (2). However, as the energy fluctuations can, in principle, be infinite, the more general distribution given by Equations (46) or (50) is required to determine the moments that characterize the actual truncated distribution. From Table II and Equation (39), it is clear that the high temperature limit is not valid for water under ambient conditions as $J_{1}=1.577$ $\ll 2$. However, this is primarily due to the intramolecular contributions to the heat capacity. For argon we find $J_{1} \approx 2$ for large parts of the phase diagram, as shown in Figure 2. For a fluid at high temperature with unit variance $\left(K_{2}=\tilde{K}_{2}=1\right)$, the partition function provides cumulants of $K_{3}=2$ and $K_{4}=6$. The same results are obtained from Equation (46) for infinite size systems.

Ruppeiner has questioned the validity of a simple entropy expansion beyond second order. ${ }^{4,11}$ This criticism is based on (at least) two aspects of the resulting pdf. First, with third order terms, the energy pdf is no longer symmetric and therefore $\langle E\rangle-E^{*}$ is no longer zero. This then violates the energy conservation and additivity laws between the system and the surrounding bath. Second, the resulting pdf is no longer covariant with respect to a change in thermodynamic coordinate 
(property). From our numerical results using the pdf described in Equation (46), we find (as expected) that, even for infinite system sizes, the mean energy and most probable energy do not coincide. However, for large system sizes, the difference between the two is fixed by the third cumulant as indicated in Equation (48). Indeed, adding an (arbitrary) fifth and sixth order term to the entropy expansion in Equation (7) does not affect this result. Therefore, we find $\beta\left(E-E^{*}\right)=\beta(E-\langle E\rangle)$ $+1 / 2 K_{3} / K_{2}$ for large systems. Hence, the properties of the distribution, determined by $\beta \delta E / K_{2}$ in Equation (46), become insensitive to whether one uses $\delta E=E-E^{*}$ or $\delta E=E-\langle E\rangle$ as the difference just corresponds to a simple shift of the pdf along the energy coordinate. This is due to the fact that while the energy fluctuations are extensive, the $K_{3} / K_{2}$ ratio is intensive, and hence $\left(\langle E\rangle-E^{*}\right) / N_{1}$ then tends to zero, as indicated in Equation (48). Consequently, the pdf obeys the criteria required for energy conservation and additivity for all but very small $\left(N_{1}<1000\right)$ systems, even though it possesses a finite skewness.

The issue of covariant behavior is more difficult to answer. A full discussion of the pdf presented here is hindered by the absence of a normalization factor. Normally, this is considered to be a simple constant. However, it is clear from the pdfs developed here that it must, at least, vary with system size and/or average energy. It is possible that any Jacobian factor arising from a change in coordinate may affect the results for very small system sizes but could lead to covariant behavior for large/infinite system sizes where the distribution resembles the properties of a simple Gaussian, as indicated by Equation (47). Alternatively, covariant behavior might be restricted to the pdf corresponding to an expansion of the entropy as a function of $N_{1}, V$, and $E$ and not the conditional pdf generated by expanding in only one thermodynamic variable.

Consequently, the current approach seems to contradict the more general conclusion by Ruppeiner (and others) that simple entropy expansions are prone to difficulties. Unfortunately, this conflict has not been resolved here. Alternative approaches for simple systems have been suggested. ${ }^{43} \mathrm{Nev}-$ ertheless, the results presented here for the energy probability distribution indicate that the first four moments and cumulants obtained from the entropy expansion agree with those obtained from the partition function. Hence, assuming this also holds for other ensembles, it appears that the present non-Gaussian distribution can be useful, at least for infinite size systems.

\section{CONCLUSIONS}

A simple general form for the probability distribution describing fluctuations in the particle number, volume, energy, and entropy has been presented. This single equation is valid for all the common ensembles of interest and has been extended to include the triplet fluctuations that describe non-Gaussian behavior. The probability distribution was developed using FST to manipulate the entropy derivatives into a convenient form and to then relate the results to experimentally available thermodynamic derivatives. It should be noted that FST is not required for this process and one could have used purely thermodynamic derivatives. However, the use of FST significantly simplifies the required manipulations. Visually, the resulting probability distribution tends towards the Gaussian result for large (infinite) system sizes. A set of four indicators for nonGaussian behavior is proposed and investigated for fluid water and argon using equation of state data. It appears that the skewness of the distribution remains finite, even for infinite systems, and that Gaussian behavior is not possible for real fluids.

\section{ACKNOWLEDGMENTS}

The project described was supported by Grant No. R01GM118719 from the National Institute of General Medical Sciences to P.E.S. and Grant No. 1F32AG048690 from the National Institute on Aging to E.A.P. The content is solely the responsibility of the authors and does not necessarily represent the official views of the National Institute of General Medical Science, the National Institute on Aging, or the National Institutes of Health. The authors would like to thank John O'Connell and George Ruppeiner for interesting comments, and the reviewers for useful comments.

\section{APPENDIX A: ENTROPY DERIVATIVES}

A specific form of the entropy derivatives is used here. In particular, we define these derivatives to be dimensionless and intensive. The first derivatives of the entropy are obtained from Equation (6) and are given by

$$
\begin{aligned}
S_{N} & \equiv \frac{1}{R}\left(\frac{\partial S}{\partial N_{1}}\right)_{V, E}=-\beta \mu_{1}, \\
S_{V} & \equiv \frac{V_{1}}{R}\left(\frac{\partial S}{\partial V}\right)_{N_{1}, E}=Z, \\
S_{E} & \equiv \frac{1}{R \beta}\left(\frac{\partial S}{\partial E}\right)_{N_{1}, V}=1 .
\end{aligned}
$$

A series of dimensionless intensive second derivatives are defined

$$
\begin{aligned}
& S_{N N} \equiv \frac{N_{1}}{R} \frac{\partial^{2} S}{\partial N_{1}^{2}}=-N_{1}\left(\frac{\partial \beta \mu_{1}}{\partial N_{1}}\right)_{V, E}, \\
& S_{V V} \equiv \frac{N_{1} V_{1}^{2}}{R} \frac{\partial^{2} S}{\partial V^{2}}=N_{1} V_{1}^{2}\left(\frac{\partial \beta p}{\partial V}\right)_{N_{1}, E} \\
& S_{E E} \equiv \frac{N_{1}}{R \beta^{2}} \frac{\partial^{2} S}{\partial E^{2}}=\frac{N_{1}}{\beta^{2}}\left(\frac{\partial \beta}{\partial E}\right)_{N_{1}, V}
\end{aligned}
$$

together with a series of cross derivatives

$$
\begin{aligned}
S_{N V} & \equiv \frac{N_{1} V_{1}}{R} \frac{\partial^{2} S}{\partial N_{1} \partial V}=-N_{1} V_{1}\left(\frac{\partial \beta \mu_{1}}{\partial V}\right)_{N_{1}, E} \\
& =N_{1} V_{1}\left(\frac{\partial \beta p}{\partial N_{1}}\right)_{V, E}, \\
S_{N E} & \equiv \frac{N_{1}}{R \beta} \frac{\partial^{2} S}{\partial N_{1} \partial E}=-\frac{N_{1}}{\beta}\left(\frac{\partial \beta \mu_{1}}{\partial E}\right)_{N_{1}, V}=\frac{N_{1}}{\beta}\left(\frac{\partial \beta}{\partial N_{1}}\right)_{V, E}, \\
S_{V E} & \equiv \frac{N_{1} V_{1}}{R \beta} \frac{\partial^{2} S}{\partial V \partial E}=\frac{N_{1} V_{1}}{\beta}\left(\frac{\partial \beta p}{\partial E}\right)_{N_{1}, V}=\frac{N_{1} V_{1}}{\beta}\left(\frac{\partial \beta}{\partial V}\right)_{N_{1}, E},
\end{aligned}
$$

where the equalities on the far right hand side are a result of Equation (A1). A similar set of dimensionless third derivatives 
can also be defined where one includes a factor of $N_{1}{ }^{2}$ and additional factors of $V_{1}$ for each volume derivative and $\beta$ for each energy derivative. For example,

$$
S_{N V E} \equiv \frac{N_{1}^{2} V_{1}}{\beta R} \frac{\partial^{3} S}{\partial N_{1} \partial V \partial E}
$$

and similarly for the other nine unique third derivatives.

\section{APPENDIX B: SECOND DERIVATIVES OF THE ENTROPY USING FST}

The dimensionless second derivatives can be expressed using just the $b$ 's provided by FST. Here we provide the relationships used to generate the results presented in Equation (14). Our approach is to develop relationships between various thermodynamic derivatives and then use the known results from FST. First, we note that

$$
\begin{aligned}
& \left(\frac{\partial \beta \mu_{1}}{\partial E}\right)_{N_{1}, V}=H_{1}\left(\frac{\partial \beta}{\partial E}\right)_{N_{1}, V}+\beta V_{1}\left(\frac{\partial p}{\partial E}\right)_{N_{1}, V}, \\
& \left(\frac{\partial \beta \mu_{1}}{\partial V}\right)_{N_{1}, E}=H_{1}\left(\frac{\partial \beta}{\partial V}\right)_{N_{1}, E}+\beta V_{1}\left(\frac{\partial p}{\partial V}\right)_{N_{1}, E}
\end{aligned}
$$

are generated from the differential of $\beta \mu_{1}\left(\beta, p, N_{1}\right)$. A series of Euler chain rule relationships are also available

$$
\begin{aligned}
& \left(\frac{\partial \beta \mu_{1}}{\partial N_{1}}\right)_{V, E}\left(\frac{\partial N_{1}}{\partial E}\right)_{\beta \mu_{1}, V}\left(\frac{\partial E}{\partial \beta \mu_{1}}\right)_{N_{1}, V}=-1, \\
& \left(\frac{\partial p}{\partial V}\right)_{N_{1}, E}\left(\frac{\partial V}{\partial E}\right)_{N_{1}, p}\left(\frac{\partial E}{\partial p}\right)_{N_{1}, V}=-1, \\
& \left(\frac{\partial \beta}{\partial V}\right)_{N_{1}, E}\left(\frac{\partial V}{\partial E}\right)_{N_{1}, \beta}\left(\frac{\partial E}{\partial \beta}\right)_{N_{1}, V}=-1
\end{aligned}
$$

together with the GCE differential, ${ }^{16}$

$$
V_{1} d \beta p=-E_{1} d \beta+d \beta \mu_{1} .
$$

The central derivative in the first two relationships of Equation (B2) can be developed using the chain rule (in $\beta$ ) to give 27

$$
\left(\frac{\partial \beta \mu_{1}}{\partial N_{1}}\right)_{V, E}=-\left(\frac{\partial \beta \mu_{1}}{\partial E}\right)_{N_{1}, V} \frac{\langle\delta E \delta E\rangle}{\left\langle\delta N_{1} \delta E\right\rangle}
$$

and $^{24}$

$$
\beta N_{1} V_{1}^{2}\left(\frac{\partial p}{\partial V}\right)_{N_{1}, E}=-S_{0}\left(b_{\varepsilon \varepsilon}+b_{1 \varepsilon} Z\right)
$$

Finally, we require the known FST based expressions for two of the derivatives appearing above ${ }^{24}$

$$
\begin{aligned}
& \frac{V_{1}}{R T}\left(\frac{\partial E_{1}}{\partial V_{1}}\right)_{T}=-\frac{b_{1 \varepsilon}+b_{11} Z}{b_{11}}, \\
& V_{1}\left(\frac{\partial p}{\partial E_{1}}\right)_{\rho_{1}}=-\frac{b_{1 \varepsilon}}{b_{\varepsilon \varepsilon} b_{11}-b_{1 \varepsilon}^{2}} .
\end{aligned}
$$

Using these relationships, all the derivatives in Equation (14) can be expressed in terms of the fluctuating quantities for the equivalent GCE.

More specifically, $S_{V E}$ was obtained from the last expression in Equation (B2) and the use of the first expression in Equation (B6); $S_{N E}$ was obtained from the first expression in Equation (B1) and the use of the last expression in Equation (B6); $S_{N N}$ was obtained from Equation (B4) and the expression for $S_{N E} ; S_{N V}$ was obtained from the last expression in Equation (B1) using the result of $S_{V E}$ and also Equation (B5); $S_{V V}$ was obtained from Equation (B3) using the results of $S_{V E}$ and $S_{N V}$; and finally $S_{E E}$ is provided directly by the last expression in Equation (D4).

\section{APPENDIX C: THIRD DERIVATIVES OF THE ENTROPY USING FST}

To obtain the third derivatives of the entropy as provided in Equations (15)-(18), we start from the second derivatives provided in Equation (14) and note that the differential of a number or energy density $(X / V)$ can be written as ${ }^{19}$

$$
d\left[\frac{\langle X\rangle}{V}\right]=\frac{\left\langle\delta X \delta N_{1}\right\rangle}{V} d \beta \mu_{1}-\frac{\langle\delta X \delta E\rangle}{V} d \beta
$$

in the GCE, i.e., using $\beta \mu_{1}$ and $\beta$ as independent variables. Extending this to include the pair fluctuations in the GCE, one finds that the required derivatives of the $b$ 's are provided by

$$
\left(\frac{\partial B_{X Y}}{\partial z}\right)_{z^{\prime}}=\frac{\left\langle\delta X \delta Y \delta N_{1}\right\rangle}{V}\left(\frac{\partial \beta \mu_{1}}{\partial z}\right)_{z^{\prime}}-\frac{\langle\delta X \delta Y \delta E\rangle}{V}\left(\frac{\partial \beta}{\partial z}\right)_{z^{\prime}}
$$

where $z^{\prime}$ indicates that all thermodynamic variables (in the $N V E$ ensemble) except for $z\left(=N_{1}, V\right.$ or $\left.E\right)$ are held constant, and $X$ and $Y$ can be $N_{1}$ or $E$. This general result can be written in terms of our reduced fluctuations such that

$$
V_{1}\left(\frac{\partial B_{X Y}}{\partial z}\right)_{z^{\prime}}=c_{X Y 1}\left(\frac{\partial \beta \mu_{1}}{\partial z}\right)_{z^{\prime}}-c_{X Y E}\left(\frac{\partial \ln \beta}{\partial z}\right)_{z^{\prime}} .
$$

The derivatives on the right hand side of Equation $(\mathrm{C} 3)$ are provided in Equations (A2), (A3), and (14), and then lead to

$$
\begin{aligned}
& N_{1} V_{1}\left(\frac{\partial B_{X Y}}{\partial N_{1}}\right)_{V, E}=-S_{0} c_{X Y n}, \\
& N_{1} V_{1}^{2}\left(\frac{\partial B_{X Y}}{\partial V}\right)_{N_{1}, E}=S_{0} c_{X Y v} \\
& \frac{N_{1} V_{1}}{\beta}\left(\frac{\partial B_{X Y}}{\partial E}\right)_{N_{1}, V}=S_{0} c_{X Y e}
\end{aligned}
$$

after noting that

$N_{1}\left(\frac{\partial E_{1}}{\partial N_{1}}\right)_{V, E}=-E_{1}, \quad\left(\frac{\partial E_{1}}{\partial V}\right)_{N_{1}, E}=0, \quad N_{1}\left(\frac{\partial E_{1}}{\partial E}\right)_{N_{1}, V}=1$.

Use of Equation (C4) provides, after some additional manipulation, the third derivatives of the entropy given in Equations (15)-(18).

\section{APPENDIX D: NON-GAUSSIAN DISTRIBUTION FUNCTION TERMS USING FST}

Here, we develop the third order term in the entropy expansion using FST to help manipulate the derivatives. If we focus on the triplet fluctuations, then only four terms survive on transforming the fluctuating variables. The contribution to $S^{(3)}$ from 
the pair fluctuations is less clean as only one of the entropy derivative combinations cancels. However, if we exclude the $\mu p T$ ensemble from consideration, i.e., only closed isobaric ensembles are considered, then the pair contributions term simplifies considerably and we find that

$$
\begin{aligned}
S^{(3)}= & F_{V V V}\left(\delta V^{E}\right)^{3}+3 F_{V V H}\left(\delta V^{E}\right)^{2} \delta H^{E}+3 F_{V H H} \delta V^{E}\left(\delta H^{E}\right)^{2} \\
& +F_{H H H}\left(\delta H^{E}\right)^{3}
\end{aligned}
$$

where,

$$
\begin{aligned}
F_{V V V} & \equiv S_{V V V}-3 S_{V V E} Z+3 S_{E E V} Z^{2}-S_{E E E} Z^{3} \\
& =S_{0}^{3} c_{s s s}+3 S_{0} b_{\varepsilon \varepsilon} \\
F_{V V H} & \equiv S_{V V E}-2 S_{E E V} Z+S_{E E E} Z^{2}=S_{0}^{3} c_{s s t}+2 S_{0} b_{1 \varepsilon} \\
F_{V H H} & \equiv S_{E E V}-S_{E E E} Z=S_{0}^{3} c_{s t t}+S_{0} b_{11} \\
F_{H H H} & \equiv S_{E E E}=S_{0}^{3} c_{t t t}
\end{aligned}
$$

and we have used the following relationships:

$$
\begin{aligned}
& s=v-e Z, \\
& t=e, \\
& n-v-e E_{1}=0 .
\end{aligned}
$$

All of the other $\left(\delta N_{1}\right)$ terms are found to be zero. This leads to the relationships provided in Equations (32) and (33).

To express the results for the c's found in Equation (D2) in terms of thermodynamic derivatives that might be provided by an equation of state, we require known results from FST. The dimensionless thermal pressure coefficient is given by ${ }^{24}$

$$
Z \frac{T}{p}\left(\frac{\partial p}{\partial T}\right)_{\rho_{1}}=Z \frac{T \alpha_{p}}{p \kappa_{T}}=-\frac{b_{1 \varepsilon}}{b_{11}}
$$

together with the density derivatives ${ }^{24}$

$$
\begin{aligned}
& \frac{p^{2}}{\rho_{1} Z^{2}} \frac{\partial^{2} \rho_{1}}{\partial p^{2}}=c_{111}-b_{11}^{2}, \\
& \frac{T p}{\rho_{1} Z} \frac{\partial^{2} \rho_{1}}{\partial T \partial p}=c_{11 \varepsilon}-b_{11}\left(1+b_{1 \varepsilon}\right),
\end{aligned}
$$

where the pressure derivatives are taken with $T$ constant and the temperature derivatives with $p$ constant. Finally, we also require the following isochoric derivatives: ${ }^{24}$

$$
\begin{aligned}
\frac{p T}{Z}\left(\frac{\partial \kappa_{T}}{\partial T}\right)_{\rho_{1}}= & c_{11 \varepsilon}-c_{111} \frac{b_{1 \varepsilon}}{b_{11}}-b_{11}, \\
Z \frac{T^{2}}{p}\left(\frac{\partial^{2} p}{\partial T^{2}}\right)_{\rho_{1}}= & \frac{b_{\varepsilon \varepsilon} b_{11}-b_{1 \varepsilon}^{2}}{b_{11}}-\frac{1}{b_{11}}\left[c_{1 \varepsilon \varepsilon}-2 c_{11 \varepsilon} \frac{b_{1 \varepsilon}}{b_{11}}\right. \\
& \left.+c_{111}\left(\frac{b_{1 \varepsilon}}{b_{11}}\right)^{2}\right], \\
\frac{T}{R}\left(\frac{\partial C_{V, m}}{\partial T}\right)_{\rho_{1}}= & -2 \frac{b_{\varepsilon \varepsilon} b_{11}-b_{1 \varepsilon}^{2}}{b_{11}}+\left[c_{\varepsilon \varepsilon \varepsilon}-3 c_{1 \varepsilon \varepsilon}\left(\frac{b_{1 \varepsilon}}{b_{11}}\right)\right. \\
& \left.+3 c_{11 \varepsilon}\left(\frac{b_{1 \varepsilon}}{b_{11}}\right)^{2}-c_{111}\left(\frac{b_{1 \varepsilon}}{b_{11}}\right)^{3}\right]
\end{aligned}
$$

for pure fluids.

The isochoric derivatives presented in Equations (11) and (D6) can be written in a more condensed form using our definition of $t$ to give

$$
\begin{aligned}
\frac{b_{t t}}{b_{11}^{2}} & =\frac{C_{V, m}}{R}, \\
\frac{c_{11 t}}{b_{11}^{2}} & =1+\frac{T}{\kappa_{T}}\left(\frac{\partial \kappa_{T}}{\partial T}\right)_{\rho_{1}}, \\
\frac{c_{1 t t}}{b_{11}^{3}} & =\frac{C_{V, m}}{R}-Z \frac{T^{2}}{p}\left(\frac{\partial^{2} p}{\partial T^{2}}\right)_{\rho_{1}}, \\
\frac{c_{t t t}}{b_{11}^{3}} & =2 \frac{C_{V, m}}{R}+\frac{T}{R}\left(\frac{\partial C_{V, m}}{\partial T}\right)_{\rho_{1}} .
\end{aligned}
$$

Then, using the relationships provided in Equations (D5), (13), and (D7), together with the fact that

$$
s=t \frac{b_{1 \varepsilon}}{b_{11}}-N_{1} \frac{C_{V, m}}{R}
$$

in Equation (33), provides the results given in Equations (35)-(39).

\section{APPENDIX E: THE ENERGY PROBABILITY DISTRIBUTION FUNCTION THROUGH FOURTH ORDER}

The intensive dimensionless fourth energy derivative of the entropy can be obtained from the third derivative provided in Equation (17) using the approach outlined in Appendix C. The result is

$S_{E E E E} \equiv \frac{N_{1}^{3}}{R \beta^{4}}\left(\frac{\partial^{4} S}{\partial E^{4}}\right)_{N_{1}, V}=S_{0}^{4}\left[d_{t t t t}-3 \frac{c_{1 t t}^{2}}{b_{11}}-3 \frac{c_{t t t}^{2}}{b_{t t}^{2}}\right]$,

where

$$
d_{t t t t}=\frac{\left\langle(\delta t)^{4}\right\rangle-3\left\langle(\delta t)^{2}\right\rangle^{2}}{\left\langle N_{1}\right\rangle}
$$

To relate this new fluctuating quantity to more common thermodynamic derivatives, we take a temperature derivative of the last expression in Equation (D6) to give

$$
6 \frac{C_{V, m}}{R}+6 \frac{T}{R}\left(\frac{\partial C_{V, m}}{\partial T}\right)_{\rho_{1}}+\frac{T^{2}}{R}\left(\frac{\partial^{2} C_{V, m}}{\partial T^{2}}\right)_{\rho_{1}}=d_{t t t t}-3 \frac{c_{1 t t}^{2}}{b_{11}} .
$$

This, together with the relationships in Equation (D7), is sufficient to express the fourth derivative of the entropy in terms of more convenient thermodynamic derivatives. Alternatively, the energy derivatives of the entropy can also be evaluated by taking multiple derivatives of Equation (6), using the chain rule for $\partial C_{V} / \partial E=\partial C_{V} / \partial T \cdot \partial T / \partial E$, and noting that $\partial E / \partial T=C_{V}$ at fixed density. Hence, FST is not required in this case and the approach becomes totally thermodynamic in nature. Both approaches give the same results.

\footnotetext{
${ }^{1}$ T. L. Hill, Statistical Mechanics (McGraw-Hill, New York, 1956).

${ }^{2}$ D. A. McQuarrie, Statistical Mechanics (Harper \& Row, New York, 1976).

${ }^{3}$ M. P. Allen and D. J. Tildesley, Computer Simulation of Liquids (Oxford University Press, USA, 1987).

${ }^{4}$ G. Ruppeiner, Rev. Mod. Phys. 67, 605 (1995).

${ }^{5}$ H. B. Callen and T. A. Welton, Phys. Rev. 83, 34 (1951).

${ }^{6}$ G. Nicolis and I. Prigogine, Proc. Natl. Acad. Sci. U. S. A. 68, 2102 (1971).
} 
${ }^{7}$ P. Colonna, N. R. Nannan, A. Guardone, and T. P. van der Stelt, Fluid Phase Equilib. 286, 43 (2009).

${ }^{8}$ M. Castier and V. F. Cabral, Fluid Phase Equilib. 334, 128 (2012).

${ }^{9}$ L. D. Landau and E. M. Lifshitz, Statistical Physics, Part I, 3rd ed., translated from the Russian by J. B. Sykes and M. J. Kearsley (Pergamon Press, Oxford, 1980).

${ }^{10}$ Y. Mishin, Ann. Phys. 363, 48 (2015).

${ }^{11}$ G. Ruppeiner, Am. J. Phys. 78, 1170 (2010).

${ }^{12}$ L. Diosi and B. Lukacs, Phys. Rev. A 31, 3415 (1985).

${ }^{13}$ J. A. Tuszynski, M. J. Clouter, and H. Kiefte, Phys. Rev. B 33, 3423 (1986).

${ }^{14}$ E. A. Ploetz, S. Karunaweera, and P. E. Smith, J. Chem. Phys. 142, 044502 (2015).

${ }^{15}$ R. F. Greene and H. B. Callen, Phys. Rev. 83, 1231 (1951).

${ }^{16}$ N. Davidson, Statistical Mechanics (McGraw-Hill, New York, 1962).

${ }^{17}$ J. G. Kirkwood and R. J. Goldberg, J. Chem. Phys. 18, 54 (1950).

${ }^{18}$ E. A. Ploetz and P. E. Smith, J. Chem. Phys. 135, 044506 (2011).

${ }^{19}$ E. A. Ploetz and P. E. Smith, Adv. Chem. Phys. 153, 311 (2013).

${ }^{20}$ E. A. Ploetz and P. E. Smith, J. Phys. Chem. B 119, 7761 (2015).

${ }^{21}$ J. G. Kirkwood and F. P. Buff, J. Chem. Phys. 19, 774 (1951).

${ }^{22}$ A. Ben-Naim, Molecular Theory of Solutions (Oxford University Press, New York, 2006).
${ }^{23}$ P. E. Smith, E. Matteoli, and J. P. O'Connell, Fluctuation Theory of Solutions: Applications in Chemistry, Chemical Engineering and Biophysics (CRC Press, Boca Raton, 2013).

${ }^{24}$ E. A. Ploetz, G. N. Pallewela, and P. E. Smith, "Fluctuation Solution Theory of Pure Fluids," J. Chem. Phys. (to be published).

${ }^{25}$ A. Munster, Classical Thermodynamics (Wiley-Interscience, London, 1970).

${ }^{26}$ P. Schofield, Proc. Phys. Soc. 88, 149 (1966).

${ }^{27}$ C. G. Gray and K. E. Gubbins, Theory of Molecular Fluids: Fundamentals (Oxford University Press, New York, 1984), Vol. 1

${ }^{28}$ H. C. Andersen, J. Chem. Phys. 72, 2384 (1980).

${ }^{29}$ W. Wagner and A. Pruss, J. Phys. Chem. Ref. Data 31, 387 (2002).

${ }^{30}$ C. Tegeler, R. Span, and W. Wagner, J. Phys. Chem. Ref. Data 28, 779 (1999).

${ }^{31}$ A. H. Harvey, A. P. Peskin, and S. A. Klein, NIST Standard Reference Database 10: NIST/ASME Steam Properties, Version 2.22 (U.S. Department of Commerce, Gaithersburg, 2008).

${ }^{32}$ E. W. Lemmon, M. L. Huber, and M. O. McLinden, REFPROP: Reference Fluid Thermodynamic and Transport Properties, Version 9.1, DLL version number 9.1, U.S. Secretary of Commerce on behalf of the United States of America: Applied Chemicals and Materials Division, 2013.

${ }^{33}$ G. Ruppeiner, Phys. Rev. A 27, 1116 (1983). 